\title{
Real-time system design environment for multi-channel high-speed data acquisition system and pattern recognition ${ }^{1}$
}

\author{
Dario B. Crosetto - 3D-Computing, Inc. \\ 900 Hideaway Pl., DeSoto, TX 75115
}

\section{E-mail: Crosetto@physics.rice.edu, Dario.Crosetto@cern.ch}

\begin{abstract}
The Design Real-Time 2.0 software environment provides the user with a set of tools to create a real-time fully programmable system which can sustain a rate of several $\mathrm{MHz}$ from multi-channel inputs, can extend processing time in a pipeline stage and can provide a result within a few hundred ns. The tool set allows the user to a) create applications of different sizes with different throughput and filtering algorithms; b) select the processor speed, internal bus width, lookup tables, number of input and output result bits for each set of data received at each channel for each algorithm execution; c) simulate a specific parallel-processing system for a given algorithm on different sets of data; d) monitor the system in real time via an RS232 interface; e) create a hardware component (FPGA or ASIC) by means of interfacing to the Electronic Design Automation (EDA) tools. The advantage of these tools is that of allowing simulation, before construction, of an entire programmable high-speed data acquisition and processing system, and selection of the processor speed, bus width and real-time algorithm which is most cost effective for a specific application where generalpurpose processors fail in speed and performance. Benefits derived from the use of the novel architecture and cost comparisons with respect to similar designs are also provided.
\end{abstract}

\section{INTRODUCTION}

The Design Real-Time 2.0 software environment for designing programmable systems capable of acquiring and processing data at a rate of several $\mathrm{MHz}$ is based on the 3DFlow architecture.

The advantage of the 3D-Flow programmable system [1], [2], [3], [4] with respect to the "hardwired" alternative systems described in the references [5], [6], [7], [8], [9], [10], [11] is in a) allowing it to adapt to unexpected operating conditions and enabling new, unforeseen physics because of its flexibility/scalability; b) simplifying the electronics in many areas (see Section V- $B$, backplane, number of boards, cost, size of the system, ease of monitoring in real-time, etc.); c) allowing extension of the processing time in a pipeline stage (see Sec. V-Al); d) providing full programmability in processing data acquired from multisensor systems at a speed not envisaged before; and e) making it possible to build software tools (the Design Real-Time) capable of creating, simulating, and verifying at the gate level before construction, entire systems of different sizes and topologies.

The 3D-Flow architecture has the above advantages because it is based on a single replicated component, while the "hardwired" system does not have the advantages because a) it would have required an exorbitant amount of effort to model each different component at the gate level, b) it would have been limited to a specific application. Similar high-speed systems in current High Energy Physics (HEP) experiments or the ones proposed for future experiments at the Large Hadron Collider at CERN, Geneva, a) make use of special hardwire trigger algorithms requiring a different circuit and a different ASIC development for each experiment, and b) use a time window for each pipeline processing stage, not to exceed the time interval between two consecutive input data. (See Sec. VA1)

The innovation is the 3D-Flow processor and system architecture which is the result of the ability to constrain an entire design to a single type of replicated component and a minimal number of different types of boards which make a system scalable, meeting the requirements of several highspeed data acquisition and processing applications.

The significance of the innovation is that it meets the requirements (see Section V-C) of the specifications for HEP applications [10], [11], [12], [13] in less development time while using the latest technology and requiring a simplified implementation at a lower cost, although it is not limited to those applications. Other commercial applications such as Positron Emission Tomography (PET), quality control in industry, high-speed data processing from multi-sensors or from high-speed communication can benefit from this innovation.

\section{THE NEED FOR PROGRAMMABILITY IN FAST REAL-TIME DATA ACQUISITION AND PROCESSING}

In commercial applications (see Fig. 1b) the demand for real-time digital video, image processing and networking is increasing. The $2.5 \mathrm{Gbps}$ optical networking products available today (and 10 Gbps available for long distances) require high-performance processing systems capable of handling Gbyte/s up to several Tbyte/s of information from multiple channels. The system should be scalable in size and also in performance as the technology level advances.

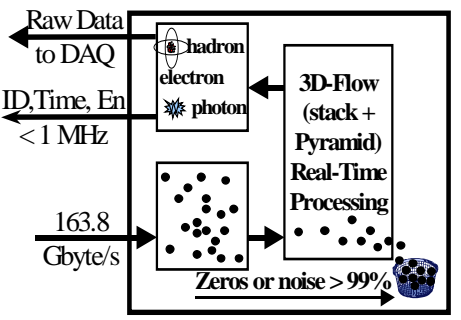

a. High Energy Physics application

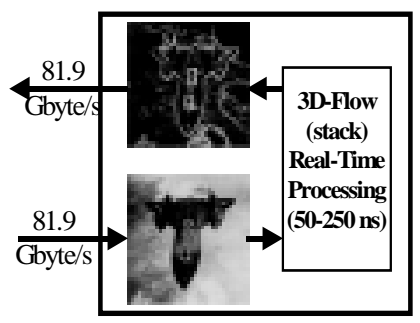

b. Commercial application
Figure 1. Performance of One 3D-Flow Crate as described in [3].

In High Energy Physics applications (see Fig. 1a) we typically have a high input data rate (of the order of 800 Gbyte/s to a few Tbyte/s) with the need to detect some specific patterns (photons/electrons, single hadrons, muons, and jets, as well as global sums of energy and missing energy). In addition, there are combinations of objects such as lepton pairs and jets with leptons or missing energy. Valid patterns which satisfy the first level of trigger algorithm criteria occur only at a rate of the order of $100 \mathrm{KHz}$ to $1 \mathrm{MHz}$.

\footnotetext{
${ }^{1}$ This material is based upon work partially funded by the Department of Energy under Grant No. DE-FG03-95ER81905
} 


\section{SOLUTION TO BREAK CURRENT SPEED BAR- RIERS IN HIGH-SPEED PROGRAMMABLE SYSTEMS}

The key element is the presence in the 3D-Flow processor and system of the Top-to-Bottom "bypass switches" (see Sec. III-A, Sec. V-Al), which remove the constraint of executing within the time interval of two consecutive input data sets, operations of

1. fetching input data;

2. exchanging with neighbors; and

3. performing eventual pattern recognition and data reduction in order to obtain a reasonable amount of reduced data that can be sent through a reasonable number of output lines.

The above feature together with the ability

1. to constrain to a single type of replicated circuit;

2. to constrain to a minimal number of different boards; and

3. to constrain to an architecture that simplifies software development and hardware assembly, and which meets the requirements of several fast real-time applications,

provides a novel processor and system architecture which breaks the current speed barriers in programmable systems.

This novel architecture feature allows for implementation of a programmable acquisition and processing system acquiring data from multi-sensors at speeds related to the processor speed in the following manner. For example, with a processor speed @ $100 \mathrm{MHz}$, the system can acquire from each channel a) 4-bit data @ $400 \mathrm{MHz}$, b) 8-bit @ $200 \mathrm{MHz}$, 16-bit@100 MHz, or 32-bit @50 MHz. The input data rate and the complexity of the real-time algorithm can change and will affect only the latency of the results.

Since the processor input Top port is 8-lines multiplexed to an internal 16-bit wide bus, the 4-bit @ $400 \mathrm{MHz}$ inputs from the sensors will require an external 1:2 multiplexer.

\section{A. Component of the technology platform}

The overall architecture is based on a single circuit, the 3D-Flow [1] Processing Element (PE), consisting of fewer than $100 \mathrm{~K}$ gates. It is technology independent and is replicated several times in a chip, on a board, and on a crate.

The 3D-Flow processor is essentially a Very Long Instruction Word (VLIW) processor. Its 128-bit-wide instruction word allows for concurrent operation (up to 26 in one cycle) of the processor's internal units: Arithmetic Logic Units (ALUs), Look Up Table memories, I/O buses, Multiply Accumulate and Divide unit (MAC/DIV), comparator units, a register file, an interface to the RS-232 serial port used to preload programs and to debug and monitor during their execution, and a program storage memory.

The high-performance I/O capability is built around four bi-directional ports (North, East, South, and West) and two mono-directional ports (Top and Bottom). The Top port receives input data and the Bottom port transmits results of calculations along successive layers. Data and results flow through the stack from the sensors to the last layer. The last layer outputs results only. (See Figure 2). A built-in pipelining capability (which complements the internal processing pipeline capability of the system) is realized using a "bypass mode," in which a processor will automatically transmit the data at its Top port to the Top port of the processor in the next layer without disturbing internal processing. The "bypass mode" is controlled in a synchronous manner by a programmable counter located on each CPU and presettable by RS-232. This feature thus provides an automatic procedure to route the incoming data to the layer free to process it.

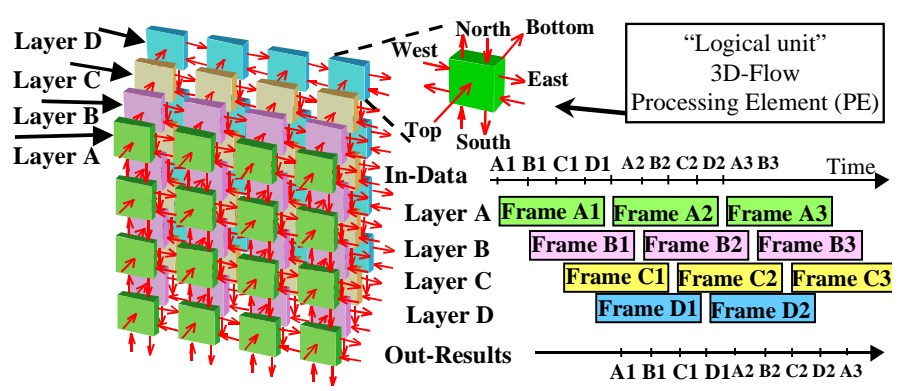

Figure 2: General scheme of the 3D-Flow architecture.

Several topologies can be built, the most common being a) a system with the same number of PEs per each layer which perform the function of pattern recognition which is called "stack," and b) a system with a decreasing number of PEs in different layers for data funneling called "pyramid." [1].

\section{B. Technology-independent 3D-Flow ASIC}

The goal of this parallel-processing architecture is to acquire multiple data in parallel and to process them rapidly, accomplishing digital filtering, pattern recognition, data exchange with neighbors, and data formatting.

Because the 3D-Flow approach is based on a single type of circuit, it is natural to keep this modularity with a single type of replicated component that does not require glue logic for its interconnection. For this reason as well as the fact that IC design advances are very rapid, it is best to retain it in IP (Intellectual Property Virtual Component) form written in generic VHDL reusable code so that can be implemented at any time using any technology. In this way it can be implemented at the last moment using the latest technology that will provide the best characteristics (low power dissipation, lower cost, smaller size, higher speed).

SOCs (System On a Chip), utilizing IPs (Intellectual Property) Virtual Components (VC), are redefining the world of electronics, as exemplified at DAC '98 conference.

Figure 3 illustrates the evolution of IC design. Even if an average component size could accommodate up to 128-PEs in the year 2001, the packaging may show an I/O pin limit, inducing one to build a component smaller than the average size.

\begin{tabular}{|c|c|c|c|c|}
\hline \multirow{3}{*}{\begin{tabular}{|c|} 
3D-Flow SOC \\
(100K gates/PE) \\
\end{tabular}} & \multicolumn{4}{|c|}{ IC Design Complexity (average component size) } \\
\hline & \multicolumn{2}{|c|}{ Current Industry } & \multicolumn{2}{|c|}{ Forecast } \\
\hline & 1997 & 1998 & 1999 & 2001 \\
\hline Logic Gates & $300 K$ & $1 \mathrm{M}$ & $5 \mathbf{M}$ & $12 \mathrm{M}$ \\
\hline Technology & $0.5 \mu \mathrm{m}$ & $0.35 \mu \mathrm{m}$ & $0.25 \mu \mathrm{m}$ & $0.18 \mu \mathrm{m}$ \\
\hline Density [gates $\left./ \mathrm{mm}^{2}\right]$ & & $\sim 14000$ & $\sim 30000$ & $\sim 65000$ \\
\hline Power [Gate/MHz] & & $700 \mathrm{nW}$ & $250 \mathrm{nW}$ & $23 \mathrm{nW}$ \\
\hline Speed & $1.4 x^{\prime} 96$ & $1.4 \times ’ 97$ & $1.4 \mathrm{x} ’ 98$ & $1.4 x^{\prime} 2000$ \\
\hline Cost/100K gates & $\$ 25$ & $\$ 5$ & $\sim \$ 2$ & $? ? ?$ \\
\hline
\end{tabular}

Figure 3. The evolution of IC design. 


\section{DESIGN REAL-TIME: THE SOFTWARE TOOLS TO INTERFACE BETWEEN APPLICATION, FPGA, AND ASIC FOR A SYSTEM DESIGNER}

Now that the 3D-Flow architecture, the component of the technology and the technology-independent ASIC have been described, the Design Real-Time software tools can be described which allow the user to design fast programmable real-time systems of different sizes, topologies, and performance (8-bit, or 16-bit wide internal buses). The steps are: a) to create a system and simulate it in software, b) using the EDA tools, to create a component in hardware, simulate, and verify each feature against the requirements of each section of the software system (e.g. stack, pyramid, real-time monitoring).

Design Real-Time is an integrated high-level design environment for the development, verification, and implementation of scalable high-speed real-time applications for which commercially available processors fail because of throughput requirements. Design Real-Time:

- $\quad$ interfaces with third-party EDA tools;

- $\quad$ is based on a single type of replicated component, the 3DFlow (PE in the form of an IP block);

- $\quad$ is technology independent because the PE, IP block can be targeted to the latest technology;

- takes the user to a higher level of abstraction and productivity gain during the design phase because of the simplicity of the 3D-Flow architecture, and the powerful tools, the set of predefined macros and the real-time algorithms available to the user;

- allows for implementation of the user's conceptual idea into the fastest programmable system at the gate level.

\section{A. 3D-Flow Design Real-Time tools}

1. create a new 3D-Flow application (called project) by varying system size, throughput, filtering algorithm, and routing algorithm, and by selecting the processor speed, lookup tables, number of input and output bits for each set of data received for each algorithm execution;

2. simulate a specified parallel-processing system for a given algorithm on different sets of data. The flow of the data can be easily monitored and traced in any single processor of the system and in any stage of the process;

3. monitor a 3D-Flow system in real-time via the RS232 interface, whether the system at the other end of the RS232 cable is real or virtual; and

4. create a 3D-Flow chip accommodating several 3D-Flow processors by means of interfacing to the Electronic Design Automation (EDA) tools.

A flow diagram guides the user through the above four phases. A system summary displays the information for a 3DFlow system created by the Design Real-Time tools.

\section{B. Interrelation between the entities in the Real- Time Design Process}

Figure 4 is separated into two sections. On the left is shown the flow of the software design and simulation process to create and simulate a 3D-Flow system, on the right is shown the System-On-a-Chip for High-speed Real-time Applications and TESting (SOC-HRATES) hardware design process. The center of the figure shows the common entities of the system:

1. the IP 3D-Flow processing element as the basic circuit to which has been constrained the functionality required by different applications;

2. a set of 3D-Flow real-time algorithms and macros organized into a library;

3. the System Monitor software package that allows the user to monitor each 3D-Flow processor of the 3D-Flow system (hardware or VPS -Virtual Processing System--), via RS-232 lines. The System Monitor (SM):

a) performs the function of a system-supervising host that loads different real-time algorithms into each processor during the initialization phase;

b) detects malfunctioning components during run-time. (A sample of data is captured at the processor speed of $80 \mathrm{MHz}$ at a preset trigger time for 8 consecutive cycles (called snap-shot), and is transferred at low speed (at the RS-232 speed of $230 \mathrm{KBaud}$ ) to the System Monitor for debugging and/or monitoring);

c) excludes malfunctioning processors with software repair by downloading into all neighbors a modified version of the standard algorithm, instructing them to ignore the offending processor.

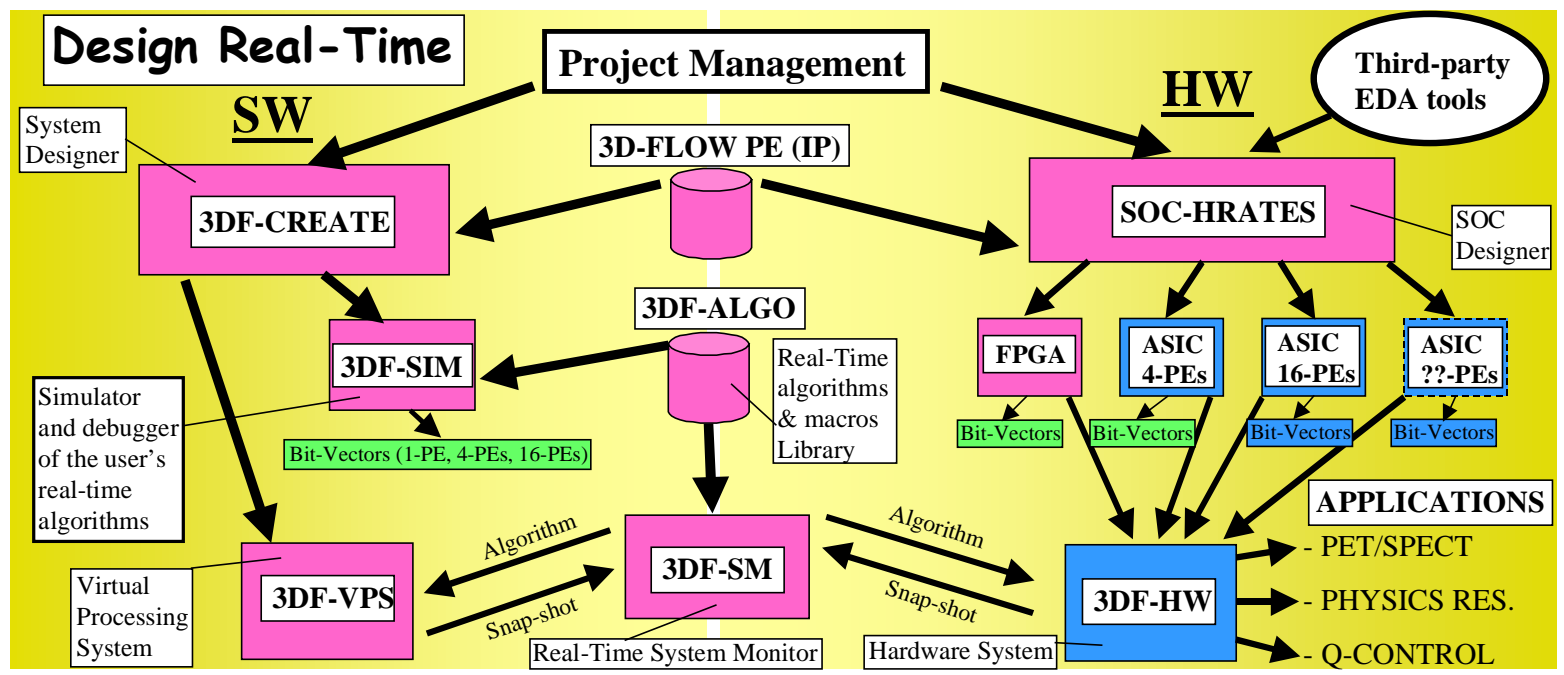

Figure 4: Interrelation between entities in the Real-Time Design Process 
The "3DF-CREATE" software module allows the user to:

1. define a 3D-Flow system of any size;

2. interconnect processors for building a specific topology with or without the channel reduction stage ("pyramid");

3. modify an existing algorithm or create a new one. The complexity of the real-time algorithms for the first levels of trigger algorithms in HEP experiments, such as the ones reported in [1], [7], [10], [11], [12], [13], [14], [15], have been examined and fewer than 10 layers (corresponding to 20 steps, each executing up to 26 operations) of $3 \mathrm{D}$-Flow processors are required;

4. create input data files to be used to test the system during the debugging and verification phase.

The "3DF-SIM" module allows for simulation and debugging of the user's system real-time algorithm and generates the "Bit-Vectors" to be compared later with the ones generated by the third-party silicon foundry tools.

The "3DF-VPS" module is the Virtual Processing System that emulates a 3D-Flow hardware system.

The right side of Figure 4 shows the hardware flow of the 3D-Flow system implementation in a System-On-a-Chip (SOC). The same common entity, the IP 3D-Flow processing element (PE), shown in the center of the figure and previously used as the behavioral model in the simulation, is now synthesized in a specific technology by using the same code.

The number of chips required for an application can be reduced by fitting several PE's into a single die. Each PE requires about $100 \mathrm{~K}$ gates and the gate density increases continually (See Fig. 3). Small 3D-Flow systems may fit into a chip. For this reason, it is also called SOC 3D-Flow. However, when an application requires the building of a 3D-Flow system that cannot be accommodated into a single chip, several chips each accommodating several 3D-Flow PEs can be interfaced with glueless logic to build a system of any size to be accommodated on a board, on a crate, or on several crates [3].

\section{Design Real-Time verification process}

The verification process of an entire 3D-Flow system can be performed down to the gate-level in the following steps:.

- The 3DF-SIM: a) extracts from the system the input data for the selected 3D-Flow processor(s) for which an equivalent hardware chip (which was targeted to a specific technology) has been created, and b) generates the BitVectors for the selected processor(s);

- The same input data and the same real-time algorithm are applied to the hardware 3D-Flow model, and the simulation is performed using the third-party tools;

- Bit-Vectors generated by the third-party tools using the hardware model are compared with the Bit-Vectors obtained by the previous software simulation (3DF-SIM);

- Discrepancies are eliminated.

\section{Results from the use of Design Real-Time}

Preliminary use of the Design Real-Time tools has made it possible to determine the parameters that led to design the data acquisition and processing system for pattern-recognition (particles in HEP experiments) described in [3] and [4], providing:

1. simulation and implementation results of a real-time system for the Level-0 trigger of LHCb [3], [4], [11] experiment at the Large Hadron Collider at CERN (which are described in the following sections of this article); and

2. the simulation and verification of the LHCb HEP Level-0 system trigger algorithm simulated using 3DF-SIM vs. the results (test pattern in the form of bit-vectors) obtained from the EDA tools from the design of

a) a single 8-bit wide internal bus 3D-Flow PE version synthesized for different FPGAs,

b) a 3D-Flow ASIC chip containing four PEs with 16-bit wide buses synthesized into a $0.5 \mu \mathrm{m}$ technology, and

c) the same four PEs into a $0.35 \mu \mathrm{m}$ ASIC technology.

Simulation has been performed, and Bit-Vectors have been compared between the system simulator (3DF-SIM) and a 3D-Flow chip implemented with $0.35 \mu \mathrm{m}$ Cell Based Array (CBA) technology at 3.3 Volts. The CBA ASIC EDA design tools show dissipation of $884 \mathrm{~mW} @$ $60 \mathrm{MHz}$ and a die size of $63.75 \mathrm{~mm}^{2}$ for a chip with 4 3D-Flow processors.

Implementation with the current technology of $0.25 \mu \mathrm{m}$ which has a gate count of $\sim 30 \mathrm{~K}$ gates per $\mathrm{mm}^{2}$ requires about $3 \mathrm{~mm}^{2}$ of silicon per PE. A chip accommodating 16 PEs requires a silicon area of about $50 \mathrm{~mm}^{2}$ in $0.25 \mu \mathrm{m}$ technology and about $25 \mathrm{~mm}^{2}$ in $0.18 \mu \mathrm{m}$ technology available next year (leading to a chip @ 1.8 Volts, 676-pin EBGA, $2.7 \mathrm{~cm} \times 2.7$ $\mathrm{cm})$. However, the latter technology dissipates ten times less power with respect to the $0.25 \mu \mathrm{m}$ version.

\section{EXAMPLE OF APPLICATIONS WHICH BENEFIT FROM DESIGN REAL-TIME TOOLS}

The benefits provided by the architecture described in this article (see full details in [3] and [4]) are applicable to similar applications which require high-speed data acquisition and processing not solvable by general-purpose microprocessors.

Current systems, however, a) use more complex hardware made of several different components and several different types of boards, b) have higher cost, c) are not easy to modify and/or to upgrade with advances in technology because their design is not technology independent, d) are not programmable and require software tools to be developed for each of them instead of having the same Design Real-Time tools for all of them.

More in general, a designer who makes use of the Design Real-Time for medical instrumentation, quality control in industry, or other applications, selects the processor speed commensurable to the a) application requirements needed and b) the technology the user wishes to use.

For the HEP application running @ $40 \mathrm{MHz}$, the processor speed of $80 \mathrm{MHz}$ was selected in order to a) easily achieve 3D-Flow instructions execution in one cycle with current lowpower CMOS technology (with the exception of the MAC/DIV instructions), and b) simplify printed circuit board construction. Assuming the selection of $80 \mathrm{MHz}$ processor speed, a multichannel acquisition and processing system can be designed and implemented with Design Real-Time which has on each channel a)32-bit data @ 40 MHz, b) 16-bit @80 MHz, 8-bit@160 MHz, or 4-bit @320 MHz. The choice for the HEP experiment was 32-bit per channel @ $40 \mathrm{MHz}$.

\section{A. Advantages of the described architecture vs. current hardwired systems}

The proposed architecture offers many advantages: flexibility, programmability, scalability, cost, software develo- 
pment simplification, simplified hardware implementation, and various other advantages in many specific applications.

The most recent questions by the trigger coordinator of the $\mathrm{LHCb}$ experiment at CERN (right after a L0 trigger implementation review) gave the opportunity to answer and to compare the advantages with alternative implementations. For example, some differences clarify the advantages such as:

\section{1) Extending processing time in a pipeline stage}

In a high-speed data acquisition and processing system such as the ones at the LHC experiments at CERN where 16to 32-bit data per channel are received every $25 \mathrm{~ns}$, a pipeline stage would not only need the time required to fetch the 32-bit input data, and to exchange the information with its neighbors (see Figure 5), but would also need the time required to reduce the data received from neighbors $(2 \times 2$, or $4 \times 4)$ in order to be able to send through the exit port every $25 \mathrm{~ns}$ a reasonable amount of reduced data through a reasonable number of lines.

The time required to reduce the data received from the neighbors depends on the level- 0 trigger algorithm. Typical operations performed are: adding values to find characteristics of possible clusters, finding local maxima, comparing with thresholds, calculating front-to-back ECAL-HCAL, etc. The operations of pattern-recognition and data moving that can be performed in $25 \mathrm{~ns}$ are very limited even with the foreseeable advances in technology.

The throughput problem posed by the need to exchange data is illustrated in Figure 5 and explained in its caption.

\section{P I P E L I N E P R O C E S S I N G}

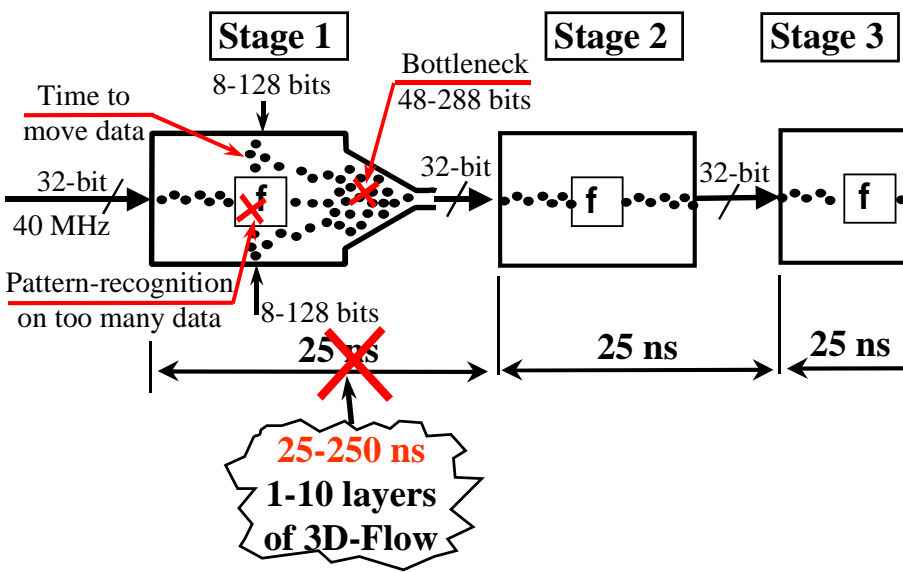

Figure 5: Stage of a pipeline system which receives input data from sensors and from neighbors every $25 \mathrm{~ns}$. To avoid the indicated bottleneck the 3D-Flow system distributes the sensors input data to different layers in a cyclic manner, thus leaving a processing time proportional to the number of layers.

The main difference between the way all other HEP groups (such as LAL, Bologna, CMS, Atlas, etc.) approach the problem and the way that $3 \mathrm{D}-\mathrm{Flow}$ architecture does, is that:

1. the former approach the implementation by splitting the trigger algorithm in pipeline stages, each not to exceed 25 ns; while

2. the 3D-Flow architecture solves the problem by replacing one pipeline stage with a stack of $3 \mathrm{D}$-Flow processors made of several processor layers (currently, in the detailed design of [3] and [4], with 1 to 10 layers) which extend the processing time for that specific stage from 25 ns up to 250 ns. (Simple algorithms use fewer layers [3]).
A design that needs to constrain each pipeline stage to 25 ns, needs to impose limitations by:

1. partitioning the problem. (The option of building a system that handles only ECAL, another that handles HCAL, is not cost effective since more electronics has to be built. The problem is just deferred to a later stage with the need to build other electronics to correlate all partial results from the ECAL, HCAL, Pad chamber, etc., subsystems, with the disadvantage of not having the possibility of using raw data from all subdetectors within a specific area in an integrated manner for better particle identification.);

2. keeping the trigger algorithm very simple. (This may not provide the best efficiency);

3. limiting the field of analysis to a small area (at the limit to a $2 \times 2$ ), with the intent to limit the number of hardware connections (Limits the efficiency);

4. designing fast electronics ("hardwired, or GaAs adder ASCIs which are not programmable but are expensive because development takes a long time and they will be outdated when they need to be used).

Trigger architectures such as the ones adopted and described in [7] and [11] from LAL and CMS (as well as the other groups such as Bologna, Atlas, etc.) have used in their solution 1) and 2), while LAL opted also for 3), CMS makes the analysis on a larger area and had developed a $200 \mathrm{MHz}$ GaAs 8-inputs 12-bit adder. Regardless, GaAs is not cost effective for common logical functions (it is more suitable for fast analog circuits, radiation-hard components, or for digital circuits@ GHz). Problems such as the one of CMS would have found a higher-performance and lower-cost solution using the 3D-Flow architecture which provides the possibility to execute algorithms requiring up to $250 \mathrm{~ns}$ and does not require special technologies such as GaAs.

If the constraint of $25 \mathrm{~ns}$ is eliminated, the user will not need to partition the problem in a section for ECAl, another for HCAL, etc., but will be able to use the raw data of a specific area from several subdetectors in an integrated manner for better particle identification.

\section{2) Software tools}

It is impractical, cost ineffective, and hard to maintain software tools based on synthesizable HDL code for a system that is made either a) of different hardwired components, or b) of a mixture of functions in FPGA and in some hardwired components. This has many disadvantages compared to the Design Real-Time which is based on the synthesizable code of a single type of PE replicated several times and interfaced with regular connections.

The great advantage of the 3D-Flow architecture to the users is that they need only to deal with 10 to 20 lines of code of the type reported in [1] and [14] each time the trigger (or pattern recognition) algorithm needs to be changed or a new application needs to be designed.

3) High risk and high cost of the "Hardwired" $25 \mathrm{~ns}$ pipeline stage implementations

Architectures such as the one described in [7], [11] by LAL and CMS have high risk or high cost because they aim to constrain each pipeline stage to $25 \mathrm{~ns}$.

The following is a series of considerations:

1. Alternative approaches such as [7], [9], [10] do not allow for future algorithm flexibility and expansion, since the fixed number of lines at 1 to $2 \mathrm{~m}$ distance of cell-to-cell communication as described in [7], Section 3, is a 
limitation for the $25 \mathrm{~ns}$ fixed time window of the highly limited architecture. Within an architecture such as the LAL, the future speed increase provided by advances in technology will provide a minimal performance increase overall, due to the major speed limitation of the information propagation time across the fixed number of lines. Moreover, if the trigger algorithm requires the analysis of more raw data, the entire system will need to be redesigned.

2. While the $2 \times 2$ algorithm [7], [10] limits the number of data exchanges, it imposes a limit, however, on event detection efficiency. The need to change the algorithm in the future might not be too remote, since for the past five years the $\mathrm{LHCb}$ experiment in the baseline had the $3 \times 3$ algorithm and large experiments such as CMS and Atlas use a $3 \times 3$ or a $4 \times 4$ trigger algorithm instead of a new $2 \times 2$ algorithm proposed for LHCb.

3. The approach of implementing the entire first-level trigger in FPGA (such as the approach in [7] by LAL) either a) must be very simple (to the detriment of trigger efficiency), or b) is very costly and difficult to handle, since the FPGA is best employed for simple combinatorial operation, but becomes very inefficient with respect to ASIC when more complex pattern recognition functions need to be implemented.

4. Similarly, more complex hardwired systems such as the one by CMS were forced to partition the tasks in small sections in order to comply with the $25 \mathrm{~ns}$ pipeline stage maximum time window, and the hardware implementation became very complex and costly (see the complexity of the crate backplane with respect to the one by the $3 \mathrm{D}$ Flow architecture which has short traces and regular nonintersecting connections), and the different types of complex board that were developed.

\section{4) FPGAs vs. ASICs}

During the phase of partitioning functions in a large design, the cost-effectiveness of implementing each function or group of functions in ASIC or in FPGA should be studied and tested.

Results of tests using different technologies and results of tests using different synthesizers which are reported in [3] and [4] show a clear advantage to implementing the front-end combinatorial logic with some pipeline buffering in FPGA, while it is more cost-effective to implement circuits aimed to process data for pattern recognition in ASIC.

\section{B. The proposed architecture simplifies the implementation}

In the 3D-Flow system, not only is the layout of the components within a board, and the boards on a crate, simplified since it has the 3D-Flow chip replicated several times and it has only a single type of board, but also because it has regular connection between components and boards.

As an example let us take the backplane and compare it with the backplane of two other implementations: LAL group [5], [6], [7], section 3.1.1, and CMS first-level trigger implementation [11] section 2.2, Figures 4 and 5. In both designs traces go from several boards to one board generating a concentration of traces higher in some areas than in other areas requiring a special construction (e.g., 13 layers of PCB required by $\mathrm{CMS}$ ).

The problem of the 3D-Flow approach needing to connect 20480 pins, which first seems to be complicated, turns out to be simpler and solvable with 6 layer PCB's. The reason for the simplicity is the original approach of the 3D-Flow that simplifies matters in many areas. A different architecture that would have to route traces connecting 20480 pins @ 400 Mbps in a "spaghetti" fashion, might require more than 30 layers.

For a clearer understanding of how the 3D-Flow architecture simplifies the backplane as compared to other implementations, Figure 6 shows the scheme of the backplane LVDS links logical layout (bottom-right) and the physical layout (bottom left) of the interconnection between neighboring processors to the North, East, West, and South.

Traces may be drawn with a controlled impedance at a value of our choice in the range of 50 to 100 Ohms. Each arrow of Figure 6 indicates a group of 320 traces. For best layout, and with the aim of eliminating all traces intersecting, one set of seven of these groups will connect pins of even connectors in one PCB layer and another set the odd connectors in another PCB layer. Each group is made of 320 non-intersecting traces of about $5 \mathrm{~cm}$ in length, laid side by side on a total width of about $32 \mathrm{~cm}$. These groups of traces will be evenly distributed on the PCB area without the formation of any high-density trace area as is the case in all alternative implementations.

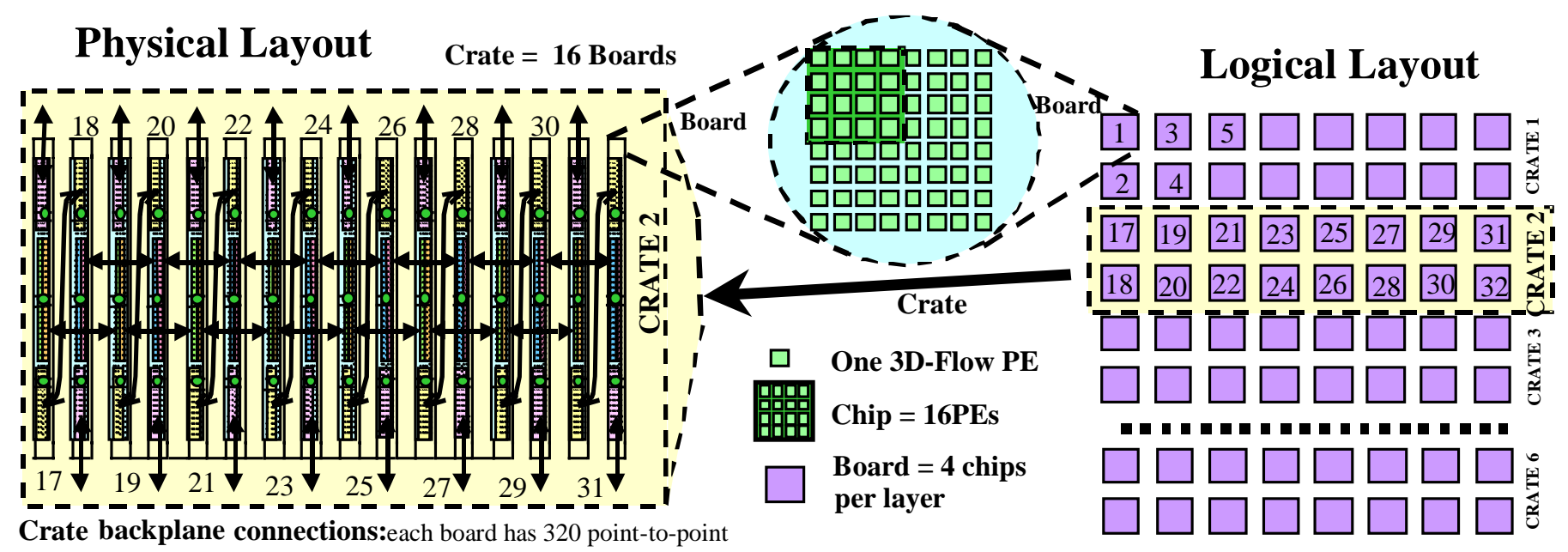

connections for LVDS links to the off-board neighboring North, East, West South chips

Figure 6: 3D-Flow backplane for neighbors PEs connections through LVDS links 


\section{Fulfil requirements necessary for Physics performance of HEP experiments}

The design of the first-level trigger algorithm of an experiment is a task of a large group of people performing many physics simulations. These simulations are usually performed in high-level language programs running on large computers and aim to find a set of parameters and an algorithm that together provide the selection of the best events containing some interesting particles (data) and reject the others.

The task of the hardware implementation, such as the 3DFlow architecture, is to take the resulting parameters and algorithm generated by the above-mentioned groups and translate them into circuits that implement the required functions at the required speed. Or in other words, translate the functions into silicon, i.e., the hardware architecture, the block scheme, the circuit, or the HDL code, the crates, the boards, the connectors, the cables, the timing, the synchronization, etc.

The parameters specify the input data rate, the number of bits per each channel, the maximum output data rate expected, the depth of the FIFO which allows for data to be buffered between two different stages of the trigger, the depth of the pipeline buffer which takes into account the time interval the input data should be kept (including cables and electronic delay) during the trigger decision time.

The trigger algorithm is the sequence of operations which needs to be performed on the input data in order to determine if the event has to be kept for further investigation or rejected.

The articles [3] and [4] describe in detail the hardware implementation of the LHCb level 0 trigger algorithm as specified in [12], (with the possibility of executing all trigger algorithms defined in the last 5 years, including the last $2 \times 2$ algorithm proposed by LAL group), which meets the requirements of the experiment.

Verification of the requirements can be done at high level by checking whether:

1. the input data rate of $40 \mathrm{MHz}$ is satisfied with a processor speed of $80 \mathrm{MHz}$;

2. the maximum number of bits from each detector channel is 23 , which is satisfied by the 32-bit/channel capability of the 3D-flow system;

3. the required output data rate is $1 \mathrm{MHz}$ for a word not to exceed 64 bits while the capability of the 3D-Flow system is a word of 64-bit @ $20 \mathrm{MHz}$ without requiring an output lookup table;

4. In 250 ns the 3D-flow system can execute (in the board designed in [3]) up to 20 steps, each one executing up to
26 operations per step which is above and beyond any

first-level algorithm foreseen in current experiments.

After this verification of the global requirements, which validates the 3D-Flow architecture against the requirement of the HEP experiment, the Design Real-Time will allow for the details to be verified by simulating any specific algorithm $(3 \times 3,2 \times 2$, etc.) from the system level to the gate level.

\section{COST/PERFORMANCE COMPARISON BETWEEN HARDWIRED SYSTEMS AND THE 3D-FLOW PROGRAMMABLE SYSTEM}

The detailed board and system design of the 3D-Flow (including a list of ICs, connectors, cables and the layout of the components on the boards) is described in [3] and [4].

To make a meaningful price comparison, a number of HEP documents quoting prices has been studied. Since the prices derived seemed low, the cost of the 3D-Flow boards has been estimated higher. The following criteria have been applied: a) 3D-Flow boards for the simpler $2 \times 2$ algorithm $\$ 4 / \mathrm{cm}^{2}$, while for the more complex $3 \times 3$ algorithm requiring more 3D-Flow chips $\$ 6.4 / \mathrm{cm}^{2}$; b) LAL-Bologna $\$ 2.7 / \mathrm{cm}^{2}$; c) CMS $\$ 3.3 / \mathrm{cm}^{2}$.

Even if the cost of the 3D-Flow board is estimated at almost twice that of the CMS boards, the 3D-Flow architecture has a definite advantage in cost-it is about three times less expensive, which will be reflected also in lower maintenance cost--in addition to its advantage in programmability, scalability, and flexibility.

LAL and Bologna boards $(36.6 \mathrm{~cm} \times 40 \mathrm{~cm})$ have been estimated at an average of $\$ 3600 /$ board. CMS large boards $(36.6 \mathrm{~cm} \times 40 \mathrm{~cm})$ have been estimated at an average of $\$ 4800 /$ board. CMS small boards ( $36.6 \mathrm{~cm}$ x $28 \mathrm{~cm}$ ) have been estimated at an average of \$3400/board.

The "3D-Flow mixed-signal processing boards," $(36.6 \mathrm{~cm}$ x $34 \mathrm{~cm}$ ) has been estimated at $\$ 5000 /$ board for the $2 \times 2 \mathrm{LAL}$ algorithm and $\$ 8000 /$ board for the complex CMS algorithm.

The cost to design a $9 \mathrm{U}$ board has been estimated at $\$ 77000$. The cost to design a backplane has been estimated at $\$ 50000$. The cost of a backplane has been estimated at $\$ 3600$. The cost of a $9 \mathrm{U}$ crate has been estimated at $\$ 9000$. Legenda:

- LAL board design (4): front-end card (248 units) - (Ref. [6] Sec. 4.1; Ref. [7] Sec. 2); ECAL summary card (28 units) (Ref. [6], Sec. 4.2.2, Ref. [7], Sec. 3.2.1); HCAL summary card (8 units) - ([6], Sec. 4.2.3; [7], Sec. 3.2.2); selection card (18 units), selection controller card (2 units) - ([6], Sec. 4.3; [7], Sec. 3.3);

- 3D-Flow board design (1): 3D-Flow mixed-signal board (96 units) - (Ref. [3], Sec. 6.1, 6,4, 6.5, and 6.6; [4], Sec. 5, and 6); Ref. [3], Sec. 6.2 for the digital board;

Table 1. Trigger cost implementation comparison between hardwired systems and the 3D-Flow programmable system.

\begin{tabular}{|c|c|c|c|c|c|c|c|c|c|c|}
\hline \multirow[t]{2}{*}{ ITEM } & \multicolumn{2}{|c|}{ Bologna 6144 Ch } & \multicolumn{2}{|c|}{ LAL Orsay 6144 Ch } & \multicolumn{2}{|c|}{ 3D-Flow 6144 Ch } & \multicolumn{2}{|c|}{ CMS 4864 Ch. } & \multicolumn{2}{|c|}{ 3D-Flow 4864 Ch } \\
\hline & $\#$ & $\begin{array}{c}\text { (boards @ } \\
\left.\$ 2.4 / \mathrm{cm}^{2}\right) \\
\text { Sys Cost } \\
{[\mathrm{K} \$]}\end{array}$ & $\#$ & $\begin{array}{c}\text { (boards @ } \\
\$ 2.4 / \mathrm{cm}^{2} \text { ) } \\
\text { Sys Cost } \\
{[\mathrm{K} \$]}\end{array}$ & $\#$ & $\begin{array}{c}(\text { boards @ } \\
\left.\$ 4 / \mathrm{cm}^{2}\right) \\
\text { Sys Cost } \\
{[\mathrm{K} \$]}\end{array}$ & \# & $\begin{array}{c}\text { (boards @ } \\
\left.\$ 3.3 / \mathrm{cm}^{2}\right) \\
\text { Sys Cost } \\
{[\mathrm{K} \$]}\end{array}$ & $\#$ & $\begin{array}{c}\text { boards @ } \\
\left.\$ 6.4 / \mathrm{cm}^{2}\right) \\
\text { Sys Cost } \\
{[\mathrm{K} \$]}\end{array}$ \\
\hline Board Design & 4 & 308 & 4 & 308 & $\overline{1}$ & 77 & 6 & 426 & 1 & 77 \\
\hline Backplane Design & 3 & 150 & 3 & 150 & 1 & 50 & 1 & 50 & 1 & 50 \\
\hline Crates inter-cabling & & & & & 40 & 9 & & & 40 & 9 \\
\hline Boards (small) & & & & & 96 & 480 & 228 & 775 & 76 & 608 \\
\hline Boards (large) & 477 & 1717 & 304 & 1094 & & & 152 & 729 & & \\
\hline Backplanes & 40 & 144 & 20 & 72 & 6 & 22 & 19 & 69 & 6 & 22 \\
\hline Crates & 40 & 360 & 20 & 180 & 6 & 54 & 19 & 171 & 6 & 54 \\
\hline Total & & \$2679K & & $\$ 1804 K$ & & $\$ 692 K$ & & $\$ 2220 \mathrm{~K}$ & & $\$ 820 K$ \\
\hline
\end{tabular}


- Bologna board design (4): front-end card (212 units) - ([8], Sec. 3.2; [9], Sec. 3, and Table 7); ECAL L0 card (208 units) - ([8], Sec. 3.2; [9], Sec. 3, and Table 7); HCAL L0 card (56 units) - ([8], Sec. 3.2; [9], Sec. 4, and Table 7); Message dispatcher card (1 unit) - ([9], Sec. 3.6, and Tb 7);

- CMS board design (6): Receiver cards (152 units); EI cards (152 units); JS cards (19 units); CEM cards (19 units); LTTC cards (19 un.); ROC cards (19 un.). Ref. [11] Sec. 2.

While the cost benefit in an experiment is considerable, even more important is the performance of the level-0 trigger, and its flexibility to accommodate future changes. The below list gives references of the features/performances. The details are described in Sections I, III, and V of this article and in the references listed in the table.

Table 2. Fast data acquisition and processing implementations: Features and Performances.

\begin{tabular}{|l|c|c|c|c|c|}
\hline ITEM & CMS & LAL & 3DF & BO & REF \\
\hline 2x2 Algorithm & & $\mathbf{X}$ & $\mathbf{X}$ & & {$[3][7]$} \\
\hline 3x3 Algorithm & $\mathbf{X}$ & & $\mathbf{X}$ & $\mathbf{X}$ & {$[\mathbf{3}][\mathbf{1 0}]$} \\
& & & & {$[\mathbf{9}][11]$} \\
\hline Fully programmable & & & $\mathbf{X}$ & & {$[\mathbf{3}]$} \\
\hline Add subsystems later & & & $\mathbf{X}$ & & {$[\mathbf{3}][4]$} \\
\hline No boundary limitation & & & $\mathbf{X}$ & & {$[3]$} \\
\hline Modular Scalable & & & $\mathbf{X}$ & & {$[3]$} \\
\hline Technology-independ. & & & $\mathbf{X}$ & & {$[3][4]$} \\
\hline
\end{tabular}

\section{CONCLUSIONS}

The Design Real-Time allows the user to create, simulate and verify the design and implementation of a programmable real-time system for which general-purpose processors are limited in speed. Its utilization has been shown to realize the advantages in application in first-level trigger in HEP (however, it is not limited to these applications). The benefits of the 3D-Flow system in HEP experiments compared to the alternative systems at all levels are:

a) SCIENTIFIC: allowing it to adapt to unexpected operating conditions and enabling new, unforeseen physics.

b) TECHNICAL: flexibility, programmability, scalability by allowing the size of the system to be expanded by cascading several crates in $x, y$ dimensions. The complexity of the algorithm ( $\mathrm{z}$ dimension) can easily be scaled to 10 times the time interval between two consecutive input data sets of 32-bit/channel using a 3DFlow processor @ $80 \mathrm{MHz}$ (corresponding to an algorithm of up to 20 steps executing up to 26 operations per step).

c) COST: at least $50 \%$ lower than the alternative proposals. The 3D-Flow architecture clearly has an advantage in cost (about three times less expensive, which will be reflected also in lower maintenance cost), even if the cost of the 3D-Flow board is estimated as almost twice that of the CMS boards. The benefit of this cost reduction is due to the innovative 3D-Flow architecture which provides also the most important advantage in system programmability, scalability, and flexibility and in simplifying its hardware implementation.

\section{ACKNOWLEDGMENTS}

I thank the SBIR office of DOE of Dr. Robert Berger, for all its support. I am grateful to A. E. Werbrouck for interesting discussions in reviewing this article. The author would like to thank also S. Conetti, B. Bonner, D. Lugari, A. Akbari, S. Bing, J. Vorgert, J. Robertazzi, and M. de Vries.

\section{REFERENCES}

[1] Crosetto, D., "High-Speed, Parallel, Pipelined, Processor Architecture for Front-End Electronics, and Method of Use Thereof.” LHCb 92-2, TRIG 96-1.

[2] Crosetto, D., "Massively Parallel-Processing System with 3D-Flow Processors." IEEE Computer Society. 0-818166322-7194, pp.355-369.

[3] Crosetto, D., "LHCb base-line level-0 trigger 3D-Flow implementation." LHCb 99-004, TRIG, 17 February 1999. http://lhcb.cern.ch/trigger/level0/3dflow/febr 17 99/lhcb99 20.pdf, http://home.att.net/ crosetto/articles/lhcb99_20.pdf

[4] Crosetto, D., "Detailed design of the digital electronics interfacing detectors, ...." LHCb 99-006, 30 March 1999. http://lhcb.cern.ch/trigger/level0/3dflow/febr_17_99/lhcb _99_006.pdf or

http://home.att.net/ crosetto/articles/lhcb_99_006.pdf

[5] D. Breton, et. Al, "An Alternative High pt Electron Trigger for LHC-B." LHCb 97-021, 3 Dec. 1997. http://lhcb.cern.ch/notes/postscript/97notes/97-021.ps

[6] The LAL LHCb group, "An Alternative High PT Electron and Hadron Trigger for LHCb." LHCb 98-058, 4 Sept. 1998.

http://hcb.cern.ch/notes/postscript/98notes/98-058.ps

[7] C. Beigbeder, et al., An Update of the $2 \times 2$ Implementation for the Level 0 Calorimeter Triggers. LHCb 99-007 29 April 1999.

http://lhcb.cern.ch/notes/postscript/99notes/99-007.ps

[8] V. Alberico, et al. The HERA-B electromagnetic pretrigger and its possible adaptations to the LHC-B Level-0 calorimeter trigger. LHCb 98-034, 10 February 1998. http://lhcb.cern.ch/notes/postscript/98notes/98-034.ps

[9] The LHCb Bologna Group. "Proposal for a Level 0 calorimeter trigger system for LHCb." LHCb 99-0013, TRIG, 29 May 1999.

http://hcb.cern.ch/notes/postscript/99notes/99-013.ps

[10] C. Beigbeder, et al., "A Joint Proposal for the Level 0 Calorimeter Triggers." LHCb 99-017, June 7, 1999. http://lhcb.cern.ch/notes/internal/postscript/99notes/99$\underline{017 . p s}$

[11] J. Lackey, et al., "CMS Calorimeter Level 1 Regional Trigger Conceptual Design." CMS note 1998/074, Nov. 13, 1998.

http://cmsdoc.cern.ch/documents/98/note98 074.pdf

[12] LHCb Technical Proposal, CERN/LHCC 98-4, 20 Feb. 1998.

[13] The Atlas Technical Proposal CERN/LHCC/94-43, 15 Dec. 1994.

[14] S. Conetti and D. Crosetto, "Implementing the Level-0 Trigger," IEEE Trans. Nucl. Sc. 43170 (1996).

[15] G. Corti, B. Cox, and D. Crosetto, "An Implementation of the L0 Muon Trigger Using the 3D-Flow system." $\mathrm{LHCb} 98-13$. 